\title{
Continuous Displacement Measurement of Soil Mass Using White Light Digital Image Analysis in Frequency Domain
}

\author{
Xinwei Yang \\ Department of traffic \\ Shijiazhuang institute of railway technology \\ Shijiazhuang, China \\ yxw1111111@126.com \\ Qifang Zhan \\ Department of traffic \\ Shijiazhuang institute of railway technology \\ Shijiazhuang, China \\ zqf221@163.com
}

\author{
Shujuan Xi \\ Department of traffic \\ Shijiazhuang institute of railway technology \\ Shijiazhuang, China \\ Xiaosuxi36@163.com \\ Xuan Ma \\ Department of traffic \\ Shijiazhuang institute of railway technology \\ Shijiazhuang, China \\ maxuan0503@126.com
}

\begin{abstract}
The displacement and deformation of soil mass is the important index in civil engineering. In this paper, white light digital image analysis in frequency domain is introduced to measure the continuous displacement of soil mass. This method has the characteristics of whole-field, non-contact measurement and the measuring system is very simple. CCD camera is used to record the images of soil mass before and after deformation. The images are digitalized and then analyzed to obtain the displacement of the specimen by using frequency domination correlation arithmetic. Comparing the experimental result with the theoretical value of displacements, the usability of this method for measuring the displacement and deformation is certified.
\end{abstract}

Keywords- soil mass; continuous displacement; white light digital image analysis in frequency domain; digital images; translation experiment.

\section{INTRODUCTION}

In civil engineering, the displacement and deformation of soil mass is always the important index in civil engineering which affects the success of the project and the security of life and property. Therefore, the measuring method for its displacement and deformation attracts the interest of the researchers[1-2].

The analysis methods for measuring displacement of soil mass include numerical method[3] and experiment method[4].

There are two major measurement methods which are commonly used which are strain gauge and displacement meter[5-6]. The advantage of two methods is that they can be used to measure the displacement and deformation of soil mass in real time and have the rapid data transmission. Meanwhile, the disadvantage of them is also obvious. First of all, it is necessary to make the measuring instrument touch the measured soil, or the instrument should be installed in the measured field. The operation progress is complex. They cannot be used in some dangerous areas;
Moreover, two methods can only be used to measure some particular points, therefore can not meet the requirement of full-field measurement and have relatively low measurement precision; Finally, the measuring instrument of two methods can only be used only one time and must be bought again next. Therefore, though these methods are suitable for a wide range, some disadvantages such as low precision, high cost and the unable whole-field measurement always affect the safety of construction engineering.

In this paper, white light digital image analysis in frequency domain [7-8] is introduced to measure the continuous displacement of soil mass. It is based on white speckle method and coherent analys is in frequency domain and has the advantages of the whole-field measurement, non-destructive measurement without coherent light source method. The method has simple light path, convenient operation, only needs a computer and a CCD camera to finish the whole record progress. The method has been already used to study the mechanical characteristics of concrete [9-10]. Therefore, the method is applicable to measure displacement and deformation of the soil mass.

\section{PRINCIPLE OF WHITE LIGHT DIGIT AL IMAGE ANALYSIS IN FREQUENCY DOMAIN}

CCD camera is used to record gray images before and after deformation and analyze them in computer to obtain the displacement information. A small region in images is considered that the displacements of the small region are equal. Let $\delta$ denote the gray distributions of one point. $\delta_{0}(x, y)$ is the location before deformation of specimen,$\delta_{1}(x, y)$ is the corresponding location after deformation and $\delta_{1}(x, y)=\delta_{0}(x+u, y+v)$ as shown in Fig. 1. $d$ is the displacement vector, $\theta$ is included angle between $d$ and $x$ axle. 


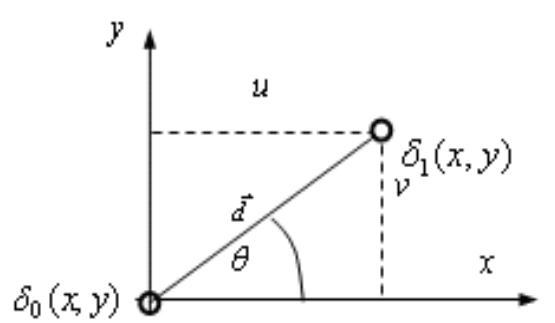

Figure 1. small region before and after deformation

$g$ is the sum of light intensity before and after deformation and then,

$$
g(x, y)=\delta_{0}(x, y)+\delta_{0}(x+d \cos \theta, y+d \sin \theta)
$$

According to Eq. 1, fourier transformation is used to obtain the spectrum:

$$
G(X, Y)=2 G_{0}(X, Y)(1+\exp (-i \pi(2 \vec{r} \cdot \vec{d}))
$$

Where,

$$
\begin{aligned}
& G_{0}(X, Y)=\mathfrak{I}\left\{\delta_{0}(x, y)\right\} \\
& =\iint \delta_{0}(x, y) \exp (-2 \pi i(x X+y Y)) d x d y=1 \\
& \mathfrak{I}\{\} \text { is fourier transformation, }(x, y) \text { is the }
\end{aligned}
$$
coordinate on object plane, $(X, Y)$ is the coordinate on spectrum plane. $\delta_{0}(x, y)$ and $G_{0}(X, Y)$ denote the gray distribution of small region in images and its Fourier transformation respectively. $\vec{r}$ is the coordinate vector on spectrum plane.

The distribution of light intensity on spectrum plane can be obtained:

$$
I=G^{*} G=4 \cos ^{2}[\pi(\vec{r} \cdot \vec{d})]
$$

According to Eq.3, when $\pi(\vec{r} \cdot \vec{d})=n \pi, I$ can be its maximum; when $\pi(\vec{r} \cdot \vec{d})=(n-1 / 2) \pi, \quad I$ is the minimum.

A series of parallel fringes can be shown in the form of image.

$$
\begin{aligned}
\vec{r} \cdot \vec{d}= & \begin{cases}n & \text { bright fringe } \\
n-\frac{1}{2} & \text { dark fringe }\end{cases} \\
& (n=0, \pm 1, \pm 2, \ldots)
\end{aligned}
$$

It is considered that the displacement of every point in small region is equal. $N$ and $N^{\prime}=N+1$ denote fringe values of adjacent fringes and then,

$r_{N} \cdot d=N, r_{N+1} \cdot d=N+1$.

So $d=(N+1)-N) /\left(r_{N+1}-r_{N}\right)$ and

$$
d=\frac{1}{\Delta r}
$$

where $\Delta r$ is the fringe separation.

Clearly, the displacement vector is vertical to the fringe direction and its magnitude can be obtained according to Eq. 5.

The imaging lens in CCD is used when images are recorded, so the real displacement $d_{T}$ is

$$
d_{T}=\frac{d}{M}=\frac{1}{M \Delta r}
$$

where $M$ is image multiple.

According to domination transformation theory, then,

$$
\Delta r=\frac{1}{\operatorname{Lccd}} \Delta K
$$

where $L c c d$ is target dimension of CCD, $\Delta K$ is pixel number between the fringes.

Substitute Eq. 7 into Eq. 6 and then,

$$
d_{T}=\frac{L c c d}{M \cdot \Delta K}
$$

$u, v$ are the components of $d_{T}$ on $x, y$ axle respectively.

\section{EXPERIMENTS}

A rectangular soil mass is selected as the specimen whose dimension is $25 \mathrm{~cm}$ length, $20 \mathrm{~cm}$ width and $2 \mathrm{~cm}$ height. The specimen is fixed on the translation stage and CCD camera is placed in front of the specimen.

Firstly, CCD camera is used to record the image of the specimen before translation. Every $1 \mathrm{~mm}$ translation, the images of the specimen after translation are recorded. The images of the specimen are input into the computer and the displacement information can be obtained by use of white light digital image analysis in frequency domain.

\section{EXPERIMENT RESULTS}

The images recorded by CCD camera are shown in Fig. 2. Because of too many images, only the four images are shown.

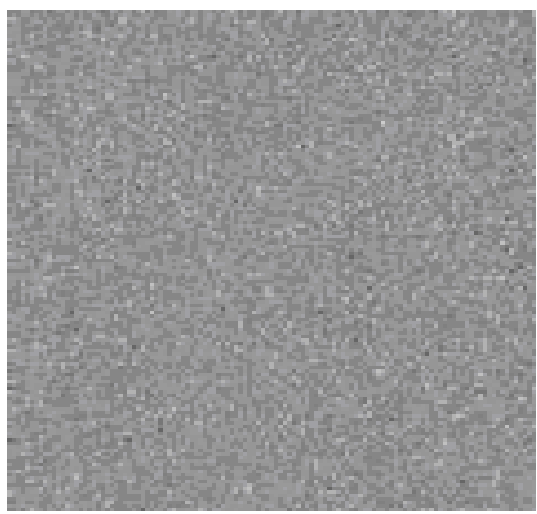

(a)before translation

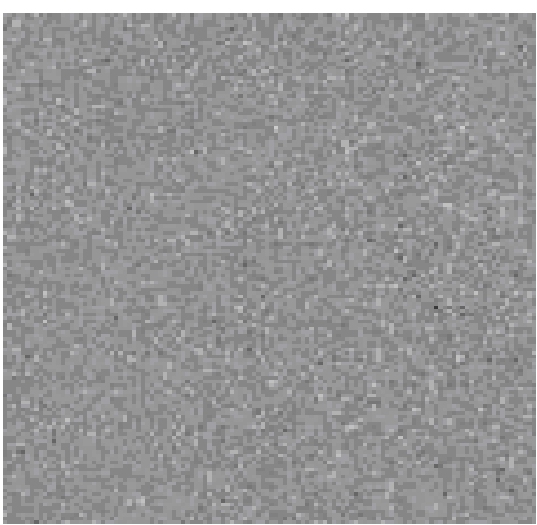

(b) $1 \mathrm{~mm}$ translation 


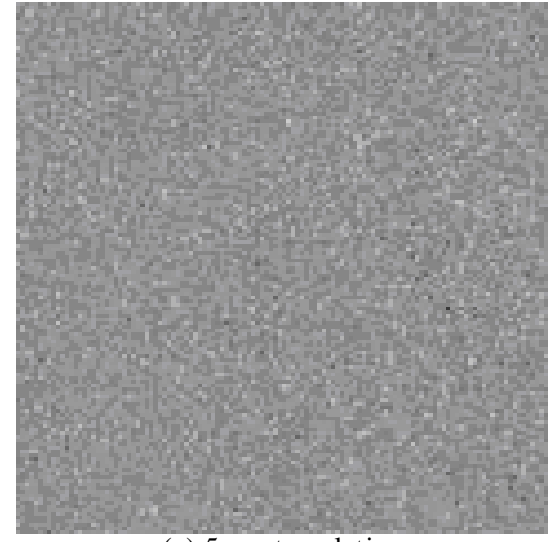

(c) $5 \mathrm{~mm}$ translation

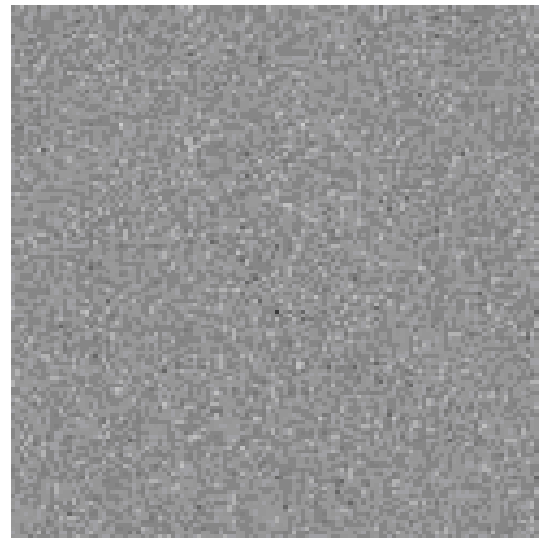

(d) $10 \mathrm{~mm}$ translation

Figure 2. the images recorded by CCD camera

A rectangle field whose length and width are $10 \mathrm{~cm}$ is chosen as the measured area and the continuous displacements which are shown in Fig. 3 can be obtained by using white light digital image analys is in frequency domain.

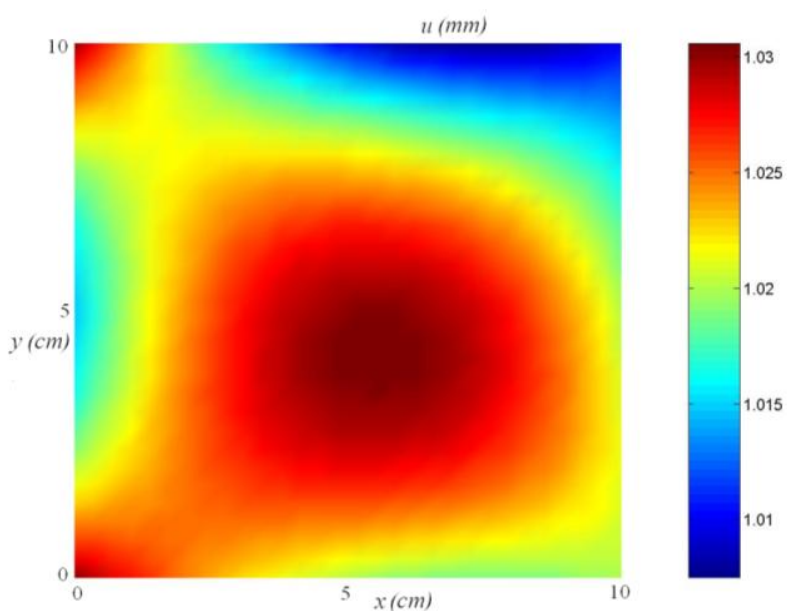

(a) $1 \mathrm{~mm}$

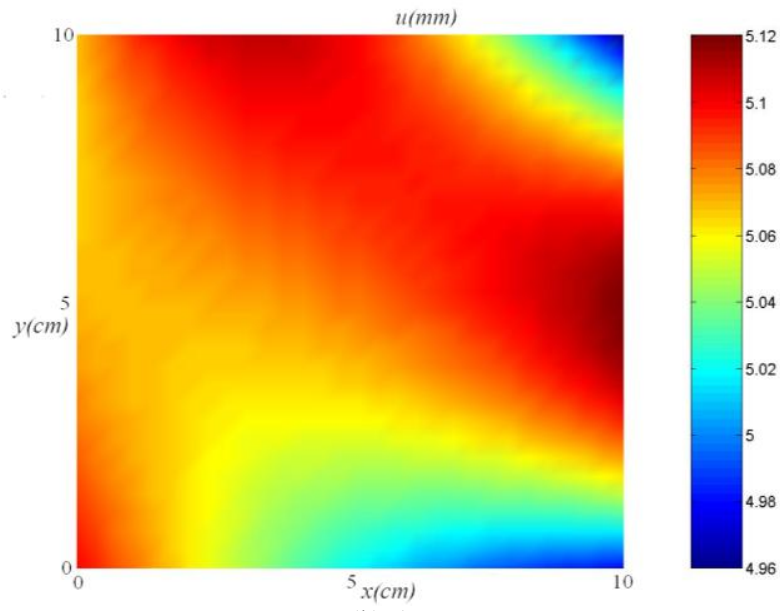

(b) $5 \mathrm{~mm}$

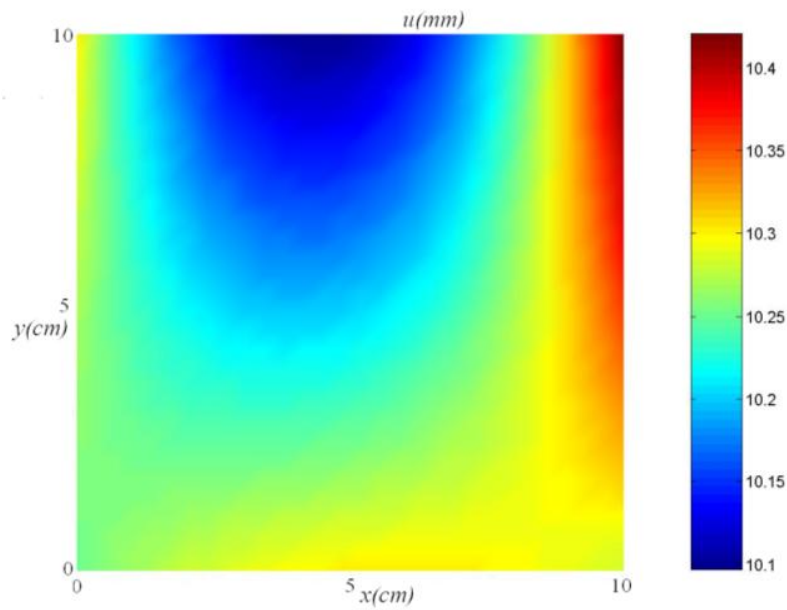

(c) $10 \mathrm{~mm}$

Figure 3. the experimental results of translation

Different color represents the values of displacement. The experimental results show that the maximum error is less than 5\% and small enough, which certifies the accuracy of the experimental results.

\section{CONCLUSIONS}

The displacement and deformation of soil mass are the important index in civil engineering. In this paper, white light digital image analysis in frequency domain is introduced to measure the continuous displacement of soil mass. This method has the characteristics of whole-field, non-contact measurement and the measuring system is very simple. The method is introduced to measure the continuous displacement of soil mass. The experimental results show that the maximum error is less than $5 \%$ and small enough, which certifies the accuracy of the experimental results. This translation experiment proves that white light digital image analys is in frequency domain can be used to measure the displacement and deformation of soil mass and further applied in the practical civil engineering.

\section{ACKNOWLEDGMENT}

The authors acknowledge the financial support of the Scientific Research Fund of Hebei Provincial Education Department, China(No. Z2014160) 


\section{REFERENCES}

[1] Li T ongyu, Guo Shibo, "Numerical analysis of tunnel-induced soil movements," Journal of Anhui Institute of Architecture and Industry, vol.21,pp. 14-21,2013

[2] Lee, CJ, "Numerical analysis of the interface shear transfer mechanism of a single pile to tunnelling in weathered residual soil", Computers and Geotechnics, vol. 42, pp. 193-203, 2012

[3] Wang Zhong-wei, Wang Haibiao, Qi Kejun, Zai Jinmin, "Summary and evaluation of experimental investigation on small strain of soil," Rock and Soil Mechanics, vol. 28, pp. 1518-1524, 2007

[4] Kong Xianjing, Jia Gexu, Zou Degao, "Deformation characteristics of rockfill at small strains," Chinese Journal of Geotechnical Engineering, vol. 23, pp. 32-37, 2001

[5] Shi Jianyong, Zhao Weibing, Lee Foukhou, "A const it utive model for anisotropic and small-strain behavior of soft clay," Rock and Soil Mechanics, vol.21,pp. 209-212,2000
[6] Kong Xianjing, Jia Gexu, Zou Degao, "Deformation characteristics of rockfill at small trains," Chniese Journal of Geotechnical Engineering, vol. 23, pp. 32-37, 2001

[7] Xu Fangyu, Ji Xinhua, "The Study in The Displacement Field Near Crack of the plexinglass by Frequency Domination Method of the White Speckle," Key Engineering Materials, vol. 306, pp. 357362,2006

[8] Ji Xinhua, Yang Xinwei, Chen Jinlong, "3D Displacement Measurement by White Light Digital Image Analysis in Frequency Domination Area," Transactions of T ianjin University, vol. 14, pp. 1-6, 2008

[9] Xinwei Yang, Honggao Man, Ruilan Tian, "Deformation Measurement of Concrete by White Light Digital Image Analysis in Frequency Domain," Applied Mechanics and Materials, vol. 2932, pp. 496-501,2010

[10] Xinwei Yang, Xiumei Jin, Ruilan Tian, "Study on Fracture Characteristics of Concrete by White Light Digitallmage Analysis in Frequency Domain," Applied Mechanics and Materials, vol. 2932, pp. 490-495, 2010 